\title{
RECEPCIÓN DE LA NOVELA ESPAÑOLA DE POSGUERRA EN LOS ESTADOS UNIDOS
}

\author{
FERNANDO GONZÁLEZ ARIZA \\ Universidad CEU San Pablo (Madrid)
}

\section{RESUMEN}

La recepción de narradores españoles en Estados Unidos durante la posguerra viene a ser una herramienta esencial para el estudio de autores y obras y su incidencia en ese país. Durante la posguerra las relaciones culturales entre los dos países fueron cambiantes: el distanciamiento hasta la mitad de los años cuarenta derivó en profundo interés durante la siguiente década y terminó con cierta indiferencia a partir de finales de los años sesenta. Presentamos así las novelas publicadas en este periodo, además de las críticas y reseñas aparecidas tanto en los principales diarios como en la prensa más especializada.

Palabras clave: Narrativa española de postguerra, Estados Unidos, Comercio del libro, Recepción crítica.

\section{RECEPTION OF THE POST-WAR SPANISH NOVEL IN THE UNITED STATES}

The critical reception of Spanish narrators in the United States through the post war period offers important data for the study of particular authors and their works, and also for their general impact in that country. During the Spanish postwar period the cultural relationships between the two countries were variable: dislike in the first years that changed in curiosity in the next decade and finally disinterest. In our study we present the novels published in this time and the reviews which appeared in the main newspapers as well as in the specialized literary reviews.

Key words: Spanish post war narrative, United States, Foreign trade of the book, Critical reception.

Hasta el primer tercio del siglo XX, las relaciones editoriales de España con el continente Americano fueron, por razones culturales y lingüísticas, prácticamente copadas por los países hispanoamericanos. A los Estados Unidos únicamente llegaron los autores españoles de más prestigio ${ }^{1}$.

\footnotetext{
${ }^{1}$ Puede consultarse el libro de Julián Amo y Charmion Shelby. La obra impresa de los intelectuales españoles en América (1936-1945). Madrid: Anabad, 1994. Aun con las limi-
} 
Otros motivos fueron la ausencia de consenso en lo que se refiere a los derechos de autor y el escaso volumen económico que movían las editoriales hasta ese momento, lo que las limitaba para hacer inversiones en el extranjero.

Sin embargo, la Guerra Civil cambió sustancialmente el panorama: el conflicto señaló al país en el mapa de las grandes confrontaciones ideológicas. La labor publicitaria de ambos bandos y el interés por parte de los diferentes países que luego se enfrentaron en la Segunda Guerra Mundial focalizó la información transmitida y provocó un interés por España hasta entonces inusitado ${ }^{2}$.

Los primeros autores que aparecieron en las librerías americanas fueron exclusivamente exiliados: Ramón J. Sender, Arturo Barea y Salvador de Madariaga. La vinculación de la España franquista a las potencias del Eje durante la guerra, unida a una ausencia efectiva de grandes novelas en esos años cuarenta, dignificó a los exiliados del mismo modo que limitó a los que permanecieron en el país o comenzaron a escribir en esos años. De cualquier manera, pocas fueron las novelas publicadas hasta 1955.

Existen dos novelas que por su situación híbrida sirvieron de eje entre los autores mencionados y la posterior llegada de novelistas no exiliados. La colmena se publica en 1953 y aunque Camilo José Cela vive en España, la novela no apareció en ese país sino que llega desde su origen argentino. Las horas finales, de Suárez Carreño (publicada por Knopf en 1954), apareció en Destino, pero el autor nació en México y aunque el resto de su vida la pasaría en España, estrictamente puede no considerarse español.

A partir de esas fechas las relaciones del gobierno de Franco se normalizan, de ser un resto del fascismo europeo pasó a considerarse un aliado anticomunista. Dicho cambio tuvo también su repercusión en el mundo del libro, pues comienzan a publicarse numerosas novelas traducidas. La primera de ellas, Los cipreses creen en Dios, fue un verdadero boom editorial. Ganó varios premios y las ediciones en Knopf se sucedieron durante bastantes años.

Además de los siguientes libros de la trilogía de Gironella, se publicó ampliamente a Juan Goytisolo, un autor que gozó muy pronto de reco-

taciones temporales que tiene; asimismo puede interesar el libro de Mercedes Caballer Dondarza. La narrativa española en la prensa estadounidense. Hallazgo, promoción, publicación y crítica (1875-1900). Madrid; Frankfurt am Main: Iberoamericana; Vervuert. 2007. Hay otra bibliografía que abarca desde los orígenes hasta la fecha de publicación, Robert Rudder, The Literature of Spain in English translation, a bibliography. New York: Ungar, 1975. Sin embargo, el autor únicamente ha consultado los fondos existentes en la Biblioteca del Congreso de Washington, muy incompleta en este asunto.

${ }^{2}$ A este respecto, y en el caso de los EE. UU., es interesante el libro de CAROLL, Peter N. y FERNÁNDEZ, James D. Facing Fascism. New York and the Spanish Civil War. New York: Museum of the City. New York University Press, 2007. 
nocido prestigio internacional y fue publicado tanto por Knopf como por Grove, dos de los sellos más literarios del país. Otros autores que llegaron entre 1955 y 1965 fueron Carmen Laforet, Ana María Matute, Ángel María de Lera, Torcuato Luca de Tena, Mercedes Salisachs o Rafael Sánchez Ferlosio. En total más de cincuenta novelas frente a las nueve que aparecieron en la década anterior. Sorprende, sin embargo, la ausencia de ciertos autores de prestigio como Miguel Delibes, Ignacio Aldecoa, Jesús Fernández Santos o Torrente Ballester, que no llegarían hasta los años setenta y ya con el membrete de clásicos.

Sin embargo, ese momento de éxito termina en los años sesenta. Aunque siguen publicándose autores españoles, y a pesar del crecimiento de la narrativa hispánica en esos años, las traducciones descienden cuantitativamente. Apenas se publican quince novelas entre 1965 y 1975 y todas ellas pertenecientes a autores ya publicados anteriormente. Se puede argumentar como motivo la llegada del 'Boom' hispanoamericano, que de algún modo pudo copar la demanda de narrativa en español por parte del público norteamericano.

A continuación presentamos el corpus de novelas publicadas en los Estados Unidos durante la posguerra. Para elaborar esta bibliografía que pensamos completa hemos consultado el ya mencionado libro de Robert Rudder. Para corregir errores y ausencias se han incluido los fondos de la New York Public Library, así como las bibliotecas de Columbia University y New York University. Entre paréntesis hemos añadido la referencia a la primera edición española.

Más adelante aparece el catálogo bibliográfico que describe las críticas literarias que obtuvieron esas novelas. Se trata de una bibliografía con interés para estudiar la recepción exterior de autores y obras concretas, asî como la incidencia general de la narrativa española en esos años.

Para la elaboración de la bibliografía crítica se han tenido en cuenta varios aspectos con la intención de servir como mapa de calidad para apreciar su recepción desde un punto de vista tanto literario como comercial. Es esencial el interés de la prensa periódica, por su rápida permeabilidad en la sociedad al dirigirse a un público amplio y por tener intención tanto crítica como informativa. Debido a la extensión del país nos hemos centrado en los grandes polos urbanos de ambas costas. Así, en la costa oeste aparecen Los Angeles Times, y el San Francisco Chronicle. Del interior del país destaca el Chicago Daily Tribune, si bien será la costa este la que posee el mayor número de rotativos y una población más culta: en la capital The Washington Post y por supuesto toda la prensa neoyorkina: The New York Times, The Nation, The New York Herald Tribune y The New Republic. Además de la prensa diaria, también se han rastreado las críticas de las revistas Time, Newsweek y The New Yorker por similares razones. 
Atendiendo también a un criterio más reposado y especialista, y con la intención añadida de llegar al mayor número de novelas reseñadas, se han incluido las revistas de crítica literaria y las dirigidas al sector del libro: Book Review, The Bookmark, The New York Review of Books, The Yale Review, Book Week, Saturday Review, Library Journal y Best Sellers.

Para facilitar la búsqueda, hemos clasificado el catálogo en diferentes apartados donde aparece el autor y la obra. Por el contrario, el corpus de obras aparecen por orden alfabético.

\section{CATÁlogo DE NOVElas ESPAÑOLAS PUBliCADAS EN LOS ESTADOS Unidos: 1945-1975}

ÁLVÁREZ DE TOLEDO, Isabel. The Strike. Trad. de William Rose. New York: Grove Press, 1971. (La huelga. Paris. Librarie du Globe, 1967).

ARRABAL, Fernando. Baal Babylon. Trad. de Richard Howard. New York: Grove Press, 1961. (Baal Babylone. Paris. Julliard, 1959).

AUB, Max. Jusep Torres Campalans. Trad. de Herbert Weinstock. New York: Doubleday, 1962. (Jusep Torres Campalans. México: Tezontle, 1958).

AYALA, Francisco. Death as a way of life. Trad. de Joan MacLean. New York: Macmillan, 1964. (Muertes de perro. Buenos Aires: Sudamericana, 1958).

—. The lamb's head. Trad. de Cecile Craig Fitzgibbons. Austin. Texas: Wiseman, 1971. (La cabeza del cordero. Buenos Aires: Losada, 1949).

BAREA, Arturo. The forging of a rebel. Trad. de Ilsa Barea. New York: Reynal \& Hitchcock, 1946. (La forja de un rebelde. Buenos Aires: Losada, 1951).

—. The broken root. Trad. de Ilsa Barea. New York: Harcourt, Brace, 1951. (La raíz rota. Buenos Aires: Santiago Rueda, 1955).

—. The forging of a rebel. Trad. de Ilsa Barea. New York: Viking Press, 1972. (La forja de un rebelde. Buenos Aires: Losada, 1951).

CELA, Camilo José. The Hive. Trad. de J. M. Cohen. New York: Farrar, Strauss \& Young, 1953. (La colmena. Buenos Aires: Emecé, 1951).

- Pabellón de reposo: Rest Home. Trad. de Herma Briffault. New York: Las Américas, 1961. (Pabellón de reposo. Madrid: Afrodisio Aguado, 1944).

—. Viaje a la Alcarria. Philip Polack (ed.). Boston: Heath, 1962. (Las botas de siete leguas. Viaje a la Alcarria. Madrid: Revista de Occidente, 1948).

-. The Family of Pascual Duarte. Trad. de Anthony Kerrigan. Boston: Atlantic-Little Brown, 1964. (La familia de Pascual Duarte. Madrid: Aldecoa, 1942).

-. Journey to the Alcarria. Trad. de Frances M. López-Morillas. Madison. University of Wisconsin Press, 1964. (Las botas de siete leguas. Viaje a la Alcarria. Madrid: Revista de Occidente, 1947)

-. Pascual Duarte and his family. Trad. de Herma Briffault. New York: Las Américas, 1965. (La familia de Pascual Duarte. Madrid: Aldecoa, 1942).

-. Mrs. Caldwell speaks to her Son. Trad. de J. S. Bernstein. Ithaca. NY: Cornell Univestity Press, 1968. (Mrs. Caldwell habla con su hijo. Barcelona: Destino, 1953).

DELIBES, Miguel. El camino. Trad. de José Amor y Vázquez \&Ruth H. Kossoff (ed.). New York: Holt, 1960. (El camino. Barcelona: Destino, 1950).

- The Path. Trad. de John and Brita Haycraft. New York: John Day Co., 1961. (El camino. Barcelona: Destino, 1950).

—. Smoke on the ground. Trad. de Alfred Johnson. Garden City. NY: Doubleday, 1972. (Las ratas. Barcelona: Destino, 1962). 
DOMINGO, Xavier. Villa Milo. Trad. de José Iglesias. New York: George Braziller, 1962. (Villa Milo. Paris. Editions du Seuil, 1961).

—. The dream of reason. Trad. de Lysander Kemp. New York: George Braziller, 1966. (Le rève de la raison. Paris. Editions du Seuil, 1963).

FERNÁNDEZ DE LA REGUERA, Ricardo. Reach for the ground. Trad. de Ilsa Barea. New York: Abelard-Schuman, 1964. (Cuerpo a tierra. Barcelona: Garbo, 1954).

FERNÁNDEZ FLÓREZ, Darío. Lola, a dark mirror. Trad. de Barbara Probst Solomon. New York: Signet Books, 1959. (Lola, espejo oscuro. Madrid: Plenitud, 1950).

GARCÍA HORTELANO, Juan. Summer Storm. Trad. de Ilsa Barea. New York: Grove Press, 1962. (Tormenta de verano. Barcelona: Seix Barral, 1962).

GIRONELLA, José María. The cypresses believe in God. Trad. de Harriet de Onis. New York: Alfred A. Knopf, 1955. (Los cipreses creen en Dios. Barcelona: Planeta, 1946).

- The cypresses believe in God. Trad. de Harriet de Onis. New York: Alfred A. Knopf, 1956. (Los cipreses creen en Dios. Barcelona: Planeta, 1946).

- Where the soul was shallow. Trad. de Anthony Kerrigan. Chicago: Henry Regnery, 1957. (Un hombre. Barcelona: Destino, 1946).

—. One million dead. Trad. de Joan MacLean. Garden City. NY: Doubleday, 1963. (Un millón de muertos. Barcelona: Planeta, 1961).

- Phantoms and fugitives: Journet to the improbable. Trad. de Terry Broch Fontseré. New York: Sheed \& Ward, 1964. (Los fantasmas de mi cerebro. Barcelona: Planeta, 1958 y Todos somos fugitivos. Barcelona: Planeta, 1961)

—. Peace after war. Trad. de Joan MacLean. New York: Alfred A. Knopf, 1969. (Ha estallado la paz. Barcelona: Planeta, 1966).

GOYTISOLO, Juan. The young assassins. Trad. de John Rust. New York: Alfred A. Knopf, 1959. (Juegos de manos. Barcelona: Destino, 1954).

—. Fiestas. Trad. de Herbert Weinstock. New York: Alfred A. Knopf, 1960. (Fiestas. Barcelona: Destino, 1958).

—. Island of women. Trad. de José Yglesias. New York: Alfred A. Knopf, 1962. (La isla. Barcelona: Seix Barral, 1961).

- The pary's over: Four attempts to define a love history. Trad. de José Yglesias. New York: Grove Press, 1966. (Fin de Fiesta. Tentativas de interpretación de una histona amorosa. Barcelona: Seix Barral, 1962).

—. Marks of identity. Trad. de Gregory Rabassa. New York: Grove Press, 1969. México: Joaquín Mortiz, 1966).

-. Count Julian. Trad. de Helen R. Lane. New York: Viking Press, 1974. (Reivindicación del conde don Julián. México: Joaquín Mortiz, 1970).

LAFORET, Carmen. Andrea. Trad. de Charles Franklin Payne. New York: Vantage Press, 1964. (Nada. Barcelona: Destino, 1945).

LASZLO, Andras. My Uncle Jacinto. Trad. de Isable Quigly. New York: Harcourt, Brace \& Co., 1958. (Mi tío Jacinto. Barcelona: José Janés, 1956).

LERA, Ángel María de. The wedding. Trad. de Stephen Kaye. New York: Dutton, 1962. (La boda. Barcelona: Destino, 1959).

—. The horns of fear. Trad. de Ilsa Barea. New York: Dutton, 1961. (Los clarines del miedo. Barcelona: Destino, 1958).

LUCA DE TENA, Torcuato. The second life of Captain Contreras. Trad. de Barnaby Conrad. Boston: Houghton Mifflin, 1960. (La segunda vida del capitán Contreras. Barcelona: Destino, 1953).

—. Another man's wife. Trad. de John Marks. New York: Alfred A. Knopf, 1965. (La mujer de otro. Barcelona: Planeta, 1962).

MADARIAGA, Salvador de. The heart of Jade. New York: Creative Age, 1944. (El corazón de piedra verde. Buenos Aires: Sudamericana, 1942). 
MARTÍN-ARTAJO, Javier. The embattled; a novel of the Spanish Civil War. Trad. de Daniel Crabb. Westminster. Maryland: The Newman Press, 1956. (No me cuente usted su caso. Madrid: Editorial Biosca, 1940).

MARTÍN DESCALZO, José. God's frontier. Trad. de Harriet de Onis. New York: Alfred A. Knopf, 1959. (La frontera de Dios. Barcelona: Destino, 1956).

MARTÍN-SANTOS, Luis. Time of Silence. Trad. de George Leeson. New York: Harcourt. Brace \& World, 1964. (Tiempo de silencio. Barcelona: Seix Barral, 1962).

MATUTE, Ana María. School of the Sun. Trad. de Elaine Kerrigan. New York: Pantheon Books, 1963. (Primera memoria. Barcelona: Destino, 1960).

—. The lost children. Trad. de Joan MacLean. New York: Macmillan, 1965. (Los hijos muertos. Barcelona: Planeta, 1958).

NÚÑEZ ALONSO, Alejandro. The purple sash. Trad. de Herma Briffault. New York: D. McKay Co., 1969. (Lazo de púrpura. Barcelona: Planeta, 1961).

ROBLES, Antonio. The refugee centaur. Trad. de Edward and Elisabeth Huberman. New York: Twayne, 1952. (El refugiado Centauro Flores, novela al día. México: Minerva, 1944).

ROYO, Rodrigo. The sun and the Snow. Trad. de Anthony Kerrigan. Chicago: Henry Regnery, 1956. (El sol y la nieve. Madrid: Talleres Gráficos CIES, 1956).

SALAZAR Y CHAPELA, Esteban. Naked in Piccadilly. Trad. de Patricia Crampton. New York and London: Abelard-Schuman, 1961. (Desnudo en Piccadilly. Buenos Aires: Losada, 1959).

SALISACHS, Mercedes. The eyes of the proud. Trad. de Delano Ames. New York: Harcourt. Brace \& Co., 1960. (Una mujer llega al pueblo. Barcelona: Planeta, 1956).

SEMPRÚN, Jorge. The Long Voyage. Trad. de Richard Seaver. New York: Grove Press, 1964. (Le grand voyage. Paris: Gallimard, 1963).

- The second death of Ramón Mercader. Trad. de Len Ortzen. New York: Grove Press, 1973. (La deuxième mort de Ramón Mercader: roman. Paris: Gallimard, 1969).

SENDER, Ramón J. A man's place. Trad. de Oliver La Farge. New York: Duell. Sloan and Pearce, 1940. (El lugar del hombre. México D.F. Quetzal, 1939).

- Dark Wedding. Trad. de Eleanor Clark. Garden City. NY: Doubleday, Doran \& Co., 1943. (Epitalamio del prieto Trinidad. México: Quetzal, 1942).

-. Chronicle of dawn. Trad. de Willard R. Trask. Garden City. NY: Doubleday, Doran \& Co., 1944. (Crónica del alba. México: Nuevo Mundo, 1942).

—. Crónica del alba. New York: F.S. Crofts \& Co., 1946. (Crónica del alba. México: Nuevo Mundo, 1942).

—. The king and the queen. Trad. de Mary Low. New York: Vanguard, 1948. (El rey y la reina. México: Editorial Jackson, 1948).

—. The Sphere. Trad. de Felix Bruno Giovanelli. New York: Hellman, Williams, 1949. (La esfera. Buenos Aires: Ediciones Siglo Veinte, 1947).

—. Los cinco libros de Ariadna. New York: Ediciones Ibérica, 1957.

-. Before noon; a novel in three parts. Trad. de W. R. Trask y F. W. Sender. Alburquerque: Univerity of New Mexico Press, 1958.

- Réquiem por un campesino español: Requiem for spanish peasant. Trad. de Elinor Randal. New York: Las Américas, 1960.

—. Seven red Sundays. Trad. de Sir Peter Chalmers Mitchell. New York: Collier Book, 1961. (Siete domingos rojos. Barcelona: Balagué, 1932).

—. La luna de los perros. New York: Las Américas, 1962.

-. The affable Hangman. Trad. de Florence Hall. New York: Las Américas, 1963. (El verdugo afable. Santiago de Chile: Nascimento, 1952).

—. La llave. New York: Las Américas, 1963.

—. La aventura equinocial de Lope de Aguirre: Antiepopeya. New York: Las Américas, 1964. 
—. Jubileo del Zócalo: Retablo conmemorativo. New York: Appleton Century Croft, 1964.

—. Tales of Cibola. Trad. de Florence Sender et al. New York: Las Américas, 1964.

- Nocturno de los 14. New York: Iberama Publishing Co., 1969.

SUÁREZ CARREÑO, José. The final hours. Trad. de Anthony Kerrigan. New York: Alfred A. Knopf, 1954. (Las horas finales. Barcelona: Destino, 1949).

SÁNCHEZ FERLOSIO, Rafael. The one day of the week. Trad. de J. M. Cohen. New York: Abelard-Schuman, 1962. (El Jarama. Barcelona: Destino, 1955).

VILALLONGA, José Luis de. The man of blood. Trad. de Hugo Charteris. New York: Simon \& Schuster, 1960. (L'homme de sang. Paris: Editions du Seuil, 1959).

—. The Pleasure Seeker. Trad. de Richard Howard. New York: Simon \& Schuster, 1962. (L’homme de plaisir. Paris: Éditions du Seuil, 1961).

\section{RECEPCIÓN CRÍTICA DE LA NOVELÍSTICA ESPAÑOLA DE POSGUERRA EN LOS ESTADOS UNIDOS}

\section{Ayala, Francisco: Death as a way of Life}

CAPOUYA, Emile. «Francisco Ayala. Death as a way of Life». Saturday Review, New York, 13-6-1964, p. 31.

GABRIEL, D. Brother. «Francisco Ayala. Death as a way of Life». Best Sellers, Washington, 15-5-1964, p. 69.

GROSS, John. «Francisco Ayala. Death as a way of Life». The New York Review of Books, New York, 9-10-1964, p. 12.

POORE, Charles. «The way of the usurper is not very tranquil». The New York Times, New York, 5-12-1964, p. 35.

RODRÍGUEZ-ALCALÁ, Hugo. «For the Power-Hungry, a dog's death waited». The New York Times Book Review, New York, 24-5-1964, p. 38.

SPECTOR, R. D. «Francisco Ayala. Death as a way of Life». Book Week, New York, 510-1964, p. 16.

\section{Barea, Arturo: Broken Root}

«Arturo Barea. Broken Root». The New Yorker, New York, 17-3-1951, p. 129.

«Behind the Lace Mantilla». Time, New York, 3-5-1951, p. 89.

BOROME, Joseph. «Arturo Barea. Broken Root». Library Journal, New York, 15-3-1951, p. 516.

BRICKELL, Herschel. «Arturo Barea. Broken Root». Saturday Review of Literature, New York, 24-4-1951, p. 17.

FULLER, Edmund. «Arturo Barea. Broken Root». Chicago Sunday Tribune, Chicago, 253-1951, p. 2.

HARRISON, J. G. «From the bookshelf». The Christian Science Monitor, Boston, 17-31951, p. 11.

HOLDEN, Richard. «Arturo Barea. Broken Root». The New Republic, New York, 23-41951, p. 21.

KRAFT, Joseph. «Return of a native». The Nation, New York, 4-7-1951, pp. 330-331.

MORTON, Frederic. «Arturo Barea. Broken Root». New York Herald Tribune, New York, 18-3-1951, p. 5.

PICKREL, Paul. «Arturo Barea. Broken Root». The Yale Review, Boston, Summer-1951, p. 765.

SENDER, Ramón J. «Arturo Barea. Broken Root». The New York Times, New York, 311-1951, p. 4. 
WEINBERGER, C. W. «Arturo Barea. Broken Root». San Francisco Chronicle, San Francisco, 4-1-1951, p. 21.

\section{Barea, Arturo: Forging of a Rebel}

«Arturo Barea. Forging of a Rebel». The New Yorker, New York, 14-12-1946, p. 145. «Spain remembered». Time, New York, 30-12-1946, p. 88.

HAMILTON, T. J. «Arturo Barea. Forging of a Rebel». The New York Times, New York, 12-8-1946, p. 4.

KRAFT, Joseph. «Arturo Barea. Forging of a Rebel». The Nation, New York, 19-6-1947, pp. 76-77.

MATTINGLY, Garrett. «Arturo Barea. Forging of a Rebel». Saturday Review of Literature, New York, 28-12-1946, p. 11.

MAYBERRY, George. «Arturo Barea. Forging of a Rebel». The New Republic, New York, 23-12-1946, p. 881.

MCDONOUGH, R. H. «Arturo Barea. Forging of a Rebel». Library Journal, New York, 15-11-1946, p. 1646.

WOLFE, B. D. «Arturo Barea. Forging of a Rebel». New York Herald Tribune, New York, 22-12-1946, p. 3.

WOODWARD, Helen. «Arturo Barea. Forging of a Rebel». Book Week, New York, 1512-1946, p. 3.

\section{Carreño, José Suárez: Final hours}

«José Suárez Carreño. Final hours». The New Yorker, New York, 23-1-1954, p. 101.

«Spanish Fatalist». Time, New York, 1-11-1954, p. 85.

BERGIN, T. G. «José Suárez Carreño. Final hours». Saturday Review, New York, 23-11954 , p. 16.

BYAM, M. S. «José Suárez Carreño. Final hours». Library Journal, New York, 15-11954, p. 145.

FLOREZ, Ángel. «José Suárez Carreño. Final hours». New York Herald Tribune, New York, 2-7-1954, p. 6.

ROLO, C. J. «José Suárez Carreño. Final hours». The Atlantic, Boston, 3-1-1954, p. 84.

SAMPSON, Paul. «Three fins life grim in Madrid». The Washington Post, Washington, 17-1-1954, p. 6.

STERN, James. «Midnight montage». The New York Times, New York, 2-7-1954, p. 26.

\section{Cela Camilo José: The family of Pascual Duarte}

«Blood hatred». Time, New York, 20-11-1964, p. 113.

«Camilo José Cela. The family of Pascual Duarte». Newsweek, New York, 16-11-1964, p. 106.

ADAMS, Mildred. «What could one expect but murder?». The New York Times Book Review, New York, 10-11-1964, p. 5.

BARRET, William. «Camilo José Cela. The family of Pascual Duarte». The Atlantic, Boston, 12-1-1964, p. 155.

CAPOUYA, Emile. «Camilo José Cela. The family of Pascual Duarte». Saturday Review, New York, 11-8-1964, p. 38.

COURTINES, Pierre. «Camilo José Cela. The family of Pascual Duarte». America, New York, 11-7-1964, p. 563.

DOOLEY, E. A. «Camilo José Cela. The family of Pascual Duarte». Best Sellers, Washington, 15-10-1964, p. 155. 
HAGEN, O. A. «Camilo José Cela. The family of Pascual Duarte». Library Journal, New York, 15-9-1964, p. 3334.

KIELY, Robert. «Cela's Spanish Ballad». The Christian Science Monitor, Boston, 14-11965, p. 7.

LAMOTT, Kenneth. «Camilo José Cela. The family of Pascual Duarte». Book Week, New York, 1-3-1965, p. 12.

WEST, Paul. «Spain between the lines». The Nation, New York, 11-2-1964, pp. 307-308.

\section{Cela, Camilo José: Journey to the Alcarria}

CARR, Raymond. «Camilo José Cela. Journey to the Alcarria». The New York Review of Books, New York, 25-11-1965, p. 24.

CINQUEMANI, F. L. «Camilo José Cela. Journey to the Alcarria». Library Journal, New York, 15-12-1964, p. 4910.

HAMILL, Peter. «Camilo José Cela. Journey to the Alcarria». Book Week, New York, 110-1965, p. 3.

\section{Cela, Camilo José: Mrs. Caldwell speaks to her son}

«Camilo José Cela. Mrs. Caldwell speaks to her son». The New Yorker, New York, 15-61968, p. 109.

CINQUEMANI, F. L. «Camilo José Cela. Mrs. Caldwell speaks to her son». Library Journal, New York, 5-1-1968, p. 1915.

DONAHUE, Francis. «Camilo José Cela. Mrs. Caldwell speaks to her son». Saturday Review, New York, 22-6-1968, p. 65.

SOURIAN, Peter. «Texture of a mind». The New York Times Book Review, New York, 26-5-1968, p. 46.

\section{Cela, Camilo José: The Hive}

«Snapshots of Madrid». Time, New York, 10-5-1953, p. 114.

BELLOW, Saul. «The evil that has many». The New York Times, New York, 27-9-1953, p. 5.

BYAM, M. S. «Camilo José Cela. The Hive». Library Journal, New York, 15-10-1953, p. 1847.

FLORES, Ángel. «Camilo José Cela. The Hive». New York Herald Tribune, New York, 27-9-1953, p. 6.

GEISMAR, Maxwell. «Camilo José Cela. The Hive». The Nation, New York, 14-11-1953, p. 404.

PICKEL, Paul. «Camilo José Cela. The Hive». The Yale Review, Boston, Winter-1954, p. XVI.

RALEIGH, J. H. «Camilo José Cela. The Hive». The New Republic, New York, 14-121953 , p. 21.

VOGLER, Lewis. «Camilo José Cela. The Hive». San Francisco Chronicle, San Francisco, 11-8-1953, p. 18.

W. M. «Camilo José Cela. The Hive». Saturday Review, New York, 24-10-1953, p. 66.

\section{Delibes, Miguel: Smoke of the ground}

ADAMS, Mildred. «Picnic on the Jarama». The New York Times, New York, 21-10-1962, p. 388.

BLOCK, Ralph. «A literary argosy sails the foam of fiction». The Washington Post, Washington, 10-7-1962, p. G7. 
LEVIN, Martin. «Miguel Delibes. Smoke of the ground». The New York Times Book Review, New York, 20-8-1972, p. 24.

\section{Domingo, Xavier: The dreams of reason}

«Of cabbages \& cops». Time, New York, 18-2-1966, p. 105.

POORE, Charles. «Our dreams of monsters must have reasons». The New York Times, New York, 3-3-1966, p. 31.

SZOGYI, Alex. «Haunted by cabbages». The New York Times Book Review, New York, 6-1966-30-6-1966, p. 27.

\section{Domingo, Xavier: Villa Milo}

«Also current». Time, New York, 3-2-1962, p. 81.

CEVASCO, G. A. «Xavier Domingo. Villa Milo». Library Journal, New York, 3-1-1962, p. 993.

FIELD, C. H. «Xavier Domingo. Villa Milo». San Francisco Chronicle, San Francisco, 22-4-1962, p. 27.

MORTON, Frederic. «A shepherd boy among exotic lambs». The New York Times Book Review, New York, 3-4-1962, p. 5.

V. H. «Xavier Domingo. Villa Milo». New York Herald Tribune, New York, 25-3-1962, p. 7.

\section{Fernández de la Reguera, Ricardo: Reach for the ground}

SYLVESTER, Harry. «Fearful warrior». The New York Times Book Review, New York, 15-11-1964, p. 58.

\section{García Hortelano, Juan: Summer storm}

BERGIN, T. G. «Juan García Hortelano. Summer storm». New York Herald Tribune, New York, 5-6-1962, p. 4.

CANTARELLA, Helene. «Of boredom on the Costa Brava». The New York Times Book Review, New York, 5-6-1962, p. 5.

JACKSON, R. B. «Juan García Hortelano. Summer storm». Library Journal, New York, 15-5-1962, p. 1915.

ROGERS, W. G. «Juan García Hortelano. Summer storm». Saturday Review, New York, 19-5-1962, p. 47.

SULLIVAN, Richard. «Qualified admiration for a first 'best' novel». Chicago Sunday Tribune, Chicago, 13-5-1962, p. 4.

\section{Gironella, José María: The cypresses believe in God}

«Mixed fiction». Time, New York, 18-4-1955, p. 116.

BERGIN, T. G. «José María Gironella. Cypresses believe in God. 2v». Saturday Review, New York, 16-4-1955, p. 14.

BOYLE, Kay. «Spain Divided». The Nation, New York, 6-11-1955, p. 506-507.

BRENAN, Gerald. «Which way for Ignatio?». The New York Times Book Review, New York, 4-10-1955, p. 5.

BYAM, M. S. «José María Gironella. Cypresses believe in God. 2v». Library Journal, New York, 15-2-1955, p. 452.

DAWEDEIT, Glendy. «Modern Spanish 'War and Peace'». The Washington Post, Washington, 4-10-1955, p. 6.

FLORES, Ángel. «José María Gironella. Cypresses believe in God. 2v». New York Herald Tribune, New York, 4-10-1955, p. 3. 
HARRISON, J. G. «José María Gironella. Cypresses believe in God. 2v». The Christian Science Monitor, Boston, 7-8-1955, p. 9.

KERRIGAN, Anthony. «Classic novel of Spain's 'social St. Vitus dance'». Chicago Sunday Tribune, Chicago, 4-10-1955, p. 6.

MCLAUGHLIN, Richard. «José María Gironella. Cypresses believe in God. 2v». Springfield Republican, Springfield, Mass., 24-4-1955, p. 8C.

ROLO, C. J. «José María Gironella. Cypresses believe in God. 2v». The Atlantic, Boston, 5-1-1955, p. 81.

VOGLER, Lewis. «José María Gironella. Cypresses believe in God. 2v». San Francisco Chronicle, San Francisco, 15-5-1955, p. 19.

WEST, A. «Books». The New Yorker, New York, 28-5-1955, p. 120.

WEST, Anthony. «José María Gironella. Cypresses believe in God. 2v». The New Yorker, New York, 28-5-1955, p. 120.

\section{Gironella, José María: One million dead}

«José María Gironella. One million dead». Newsweek, New York, 21-10-1963, p. 117.

BYAM, M. S. «José María Gironella. One million dead». Library Journal, New York, 12 1-1963, p. 4663.

CURLEY, Thomas. «The arena is Spain». The New York Times Book Review, New York, 20-10-1963, p. 4.

PAYNE, Robert. «José María Gironella. One million dead». Saturday Review, New York, 30-11-1963, p. 33.

WEST, Anthony. «José María Gironella. One million dead». The New Yorker, New York, 29-2-1964, p. 130.

\section{Gironella, José María: Peace after war}

PAYNE, Robert. «José María Gironella. Peace after war». Saturday Review, New York, 17-5-1969, p. 49.

GALLAGHER, David. «The first three years of the Thirty Years' Peace». The New York Times Book Review, New York, 6-1-1969, p. 4.

LANCOUR, Harold. «Peace after war». Library Journal, New York, 4-1-1969, p. 1518.

RYAN, F. L. «Peace after war». Best Sellers, Washington, 5-1-1969, p. 41.

DAVIS, T. N. «Peace after war». America, New York, 19-4-1969, p. 482.

Gironella, José María: Phantoms and fugitives: journeys to the improbable

COLES, Robert. «José María Gironella. Phantoms and fugitives: journeys to the improbable». Book Week, New York, 26-7-1964, p. 10.

CURLEY, Thomas. «Out of dreams, a petrified sea». The New York Times Book Review, New York, 3-1-1964, p. 4.

DAVIS, T. N. «José María Gironella. Phantoms and fugitives: journeys to the improbable». America, New York, 22-2-1964, p. 265.

NORDBERG, R. B. «José María Gironella. Phantoms and fugitives: journeys to the improbable». Best Sellers, Washington, 3-1-1964, p. 414.

\section{Gironella, José María: Where the soil was shallow}

ADAMs, Mildred. «Senorito's Progress». The New York Times, New York, 17-11-1957, p. 53.

BERGIN, T. G. «José María Gironella. Where the soil was shallow». Saturday Review, New York, 10-12-1957, p. 56. 
L. V. «José María Gironella. Where the soil was shallow». San Francisco Chronicle, San Francisco, 27-10-1957, p. 39.

\section{Goytisolo, Juan: Count Julian}

DOUGHERTY, Dru. «Juan Goytisolo. Count Julian». Library Journal, New York, 15-41974, p. 1149.

FUENTES, Carlos. «Juan Goytisolo. Count Julian». The New York Times Book Review, New York, 5-5-1974, p. 5.

PRITCHETT, V. S. «Juan Goytisolo. Count Julian». The New Yorker, New York, 10-71974, p. 173.

PROBS SOLOMON, Barbara. "As through a child's eyes». The Nation, New York, 3-11975, pp. 250-252.

THOMPSON, R. J. «Juan Goytisolo. Count Julian». Best Sellers, Washington, 15-6-1974, p. 143.

WOOD, Michael. «Juan Goytisolo. Count Julian». The New York Review of Books, New York, 8-8-1974, p. 40.

\section{Goytisolo, Juan: Fiestas}

DEMPSEY, David. «No silver lining». The New York Times Book Review, New York, 155-1960, p. 5.

GORN, L. H. «Juan Goytisolo. Fiestas». San Francisco Chronicle, San Francisco, 6-101960, p. 27.

SENDER, Ramón J. «Juan Goytisolo. Fiestas». Saturday Review, New York, 6-11-1960, p. 35.

WICKENDEN, Dan. «Juan Goytisolo. Fiestas». New York Herald Tribune, New York, 65-1960, p. 3.

\section{Goytisolo, Juan: Island of women}

CANTARELLA, Helene. «Juan Goytisolo. Island of women». The New York Times Book Review, New York, 18-3-1962, p. 5.

CINQUEMANI, F. L. «Juan Goytisolo. Island of women». Library Journal, New York, 2-1-1962, p. 784.

KIRSCH, Robert. «Suden death suggested for 'A long happy life'». Los Angeles Times, Los Angeles, 3-1-1962, p. 4.

PETITCLERC, Denne. «Juan Goytisolo. Island of women». San Francisco Chronicle, San Francisco, 22-4-1962, p. 29.

R. S. P. «Juan Goytisolo. Island of women». Springfield Republican, Springfield, Mass., 29-4-1962, p. 2D.

W. G. R. «Juan Goytisolo. Island of women». New York Herald Tribune, New York, 48-1962, p. 7.

\section{Goytisolo, Juan: Marks of identity}

KLAIDMAN, Stephen. «Poetic wrapping». The Washington Post, Washington, 28-7-1969, p. 4.

PROBS SOLOMON, Barbara. «As through a child's eyes». The Nation, New York, 3-11975, pp. 250-252.

Goytisolo, Juan: The party's over: four attempts to define a love story

COLEMAN, Alexander. «Black Vision». The New York Times Book Review, New York, 22-1-1967, p. 4. 
KARLEN, Arno. «Definitions of love». The Nation, New York, 5-1-1967, pp. 568-569. MAURER, Robert. «Juan Goytisolo. The party's over: four attempts to define a love story». Book Week, New York, 2-5-1967, p. 6.

TRACY, Honor. «Juan Goytisolo. The party's over: four attempts to define a love story». The New Republic, New York, 4-8-1967, p. 29.

WOLF, J. H. «Juan Goytisolo. The party's over: four attempts to define a love story». Library Journal, New York, 15-1-1967, p. 256.

\section{Goytisolo, Juan: Young assassins}

«Juan Goytisolo. Young assassins». Time, New York, 16-2-1959, p. 102.

ADAMS, Mildred. «Anything to get attention». The New York Times, New York, 15-21959, p. 4.

DEMPSEY, David. «Juan Goytisolo. Young assassins». Saturday Review, New York, 142-1959, p. 28.

HEALEY, R. C. «Juan Goytisolo. Young assassins». New York Herald Tribune, New York, 19-4-1959, p. 11.

MANN, C. W. «Juan Goytisolo. Young assassins». Library Journal, New York, 3-1-1959, p. 777.

\section{Laszlo, Andras: My Uncle Jacinto}

PIPPET, Roger. «Rheumatic Matador». The New York Times Book Review, New York, 1910-1958, p. 56.

\section{Lera, Ángel María de: The horns of fear}

CONRAD, Barnaby. «Ángel María de Lera. The horns of fear». Saturday Review, New York, 8-5-1961, p. 14.

DRESDEN, Donald. «The moment of truth». The New York Times Book Review, New York, 16-7-1961, p. 22.

H. B. H. «Ángel María de Lera. The horns of fear». Springfield Republican, Springfield, Mass., 5-6-1961, p. 5D.

HARRISON, W. K. «Ángel María de Lera. The horns of fear». Library Journal, New York, 8-1-1961, p. 2682.

W. E. P. «Ángel María de Lera. The horns of fear». San Francisco Chronicle, San Francisco, 9-3-1961, p. 24.

\section{Lera, Ángel María de: The wedding}

MERCHANT, Norris. «A literary argosy sails the foam of fiction». The Washington Post, Washington, 10-7-1962, p. G7.

\section{Luca de Tena, Torcuato: The second life of Captain Contreras}

KIRSCH, Robert R. «Torcuato Luca de Tena. The second life of Captain Contreras». Los Angeles Times, Los Angeles, 14-10-1960, p. 5.

\section{Madariaga, Salvador de: Heart of jad}

BEALS, Carleton. «Salvador de Madariaga. Heart of jad». Saturday Review of Literature, New York, 25-3-1944, p. 9.

KENNEDY, Leo. «Salvador de Madariaga. Heart of jad «, Book Week, New York, 4-21944, p. 4.

LERMAN, Leo. «Salvador de Madariaga. Heart of jad «, The New York Times, New York, 4-2-1944, p. 5. 
OWEN, Margaret. «Salvador de Madariaga. Heart of jad «, Library Journal, New York, 15-2-1944, p. 160.

WOLFE, B. D. «Salvador de Madariaga. Heart of jad «, New York Herald Tribune, New York, 4-9-1944, p. 2.

\section{Martín Descalzo, José Luis: God's frontier}

«José Luis Martín Descalzo. God's frontier». Springfield Republican, Springfield, Mass., 25-10-1959, p. 3E.

FLORIT, Eugenio. «José Luis Martín Descalzo. God's frontier». Saturday Review, New York, 26-9-1959, p. 19.

HALL, Vernon. «José Luis Martín Descalzo. God's frontier». New York Herald Tribune, New York, 11-1-1959, p. 3.

HEISER, H. C. «José Luis Martín Descalzo. God's frontier». Library Journal, New York, 9-12-1959, p. 2662.

REYNOLDS, Horace. «Castilian echo». The New York Times Book Review, New York, 18-10-1959, p. 48.

\section{Martín Santos, Luis: Time of silence}

CURLEY, Thomas. «Man lost in Madrid». The New York Times Book Review, New York, 29-11-1964, p. 57.

GAINES, E. J. «Luis Martín-Santos. Time of Silence». Library Journal, New York, 11-11964, p. 4388.

HAYES, Richard. «Luis Martín-Santos. Time of Silence». Book Week, New York, 11-81964, p. 8.

WEST, Paul. «Luis Martín-Santos. Time of Silence». The Nation, New York, 11-2-1964, p. 307.

\section{Matute, Ana María: School of the Sun}

ADAMS, Mildred. «Two adolescents bent on evil». The New York Times Book Review, New York, 21-4-1963, p. 4.

MORALES, J. L. «Ana María Matute. School of the Sun». Best Sellers, Washington, 154-1963, p. 28.

WEST, Anthony. «Ana María Matute. School of the Sun». The New Yorker, New York, 10-5-1963, p. 182.

YIVISAKER, Miriam. «Ana María Matute. School of the Sun». Library Journal, New York, 15-5-1963, p. 2027.

Matute, Ana María: The Lost Children

JANEWAY, Elisabeth. «Spain: 'the shutters are down'». Christian Science Monitor, Boston, 20-5-1965, p. 7.

\section{Royo, Rodrigo: The sun and the snow}

MATTHEWS, Herbert L. «With the Blue Division». The New York Times, New York, 205-1956, p. 276.

\section{Salazar y Chapela, Enrique: Naked in Piccadilly}

LEVIN, Martin. «Enrique Salazar y Chapela. Naked in Piccadilly». The New York Times Book Review, New York, 18-2-1962, p. 21. 


\section{Salisachs, Mercedes: The eyes of the proud}

«Mercedes SalisachsThe eyes of the proud». The Bookmark, Albany, N. Y. 12-1-1960, p. 68.

BERGIN, T. G. «Mercedes Salisachs. The eyes of the proud». New York Herald Tribune, New York, 30-10-1960, p. 6.

JONES, F. N. «Mercedes Salisachs. The eyes of the proud». Library Journal, New York, 15-9-1960, p. 3105.

MOYANO, María Clara. «Mercedes Salisachs. The eyes of the proud». The Washington Post, Washington, 30-10-1960, p. 7.

BARKHAM, John. «Collective guilt». The New York Times Book Review, New York, 1311-1960, p. 46.

«Mercedes Salisachs. The eyes of the proud». Time, New York, 17-10-1960, p. 112.

\section{Semprún, Jorge: The long voyage}

CAPOUYA, Emile. «Jorge Semprún. The long voyage». Saturday Review, New York, 59-1964, p. 50.

KAUFFMANN, Stanley. «Jorge Semprún. The long voyage». The New York Review of Books, New York, 18-5-1964, p. 7.

MITGANG, Herbert. «The Nazi German stain». The New York Times, New York, 29-41964, p. 39.

NYREN, Dorothy. «Jorge Semprún. The long voyage». Library Journal, New York, 155-1964, p. 2116.

ROUDIEZ, L. S. «A free man rode also in the boxcar». The New York Times Book Review, New York, 5-3-1964, p. 5.

SPECTOR, R. D. «Jorge Semprún. The long voyage». Book Week, New York, 5-10-1964, p. 16.

WEST, Anthony. «Jorge Semprún. The long voyage». The New Yorker, New York, 8-81964, p. 87.

\section{Semprún, Jorge: The second death of Ramon Mercader}

«Jorge Semprún. The second death of Ramon Mercader». The New Yorker, New York, 17-1974, p. 75.

JUDSON, Horace. «Spies and surfaces». Time, New York, 22-10-1973, p. 107.

KELLY, J. Y. «Jorge Semprún. The second death of Ramon Mercader». Library Journal, New York, 15-12-1973, p. 3651.

LAUT, S. J. «Jorge Semprún. The second death of Ramon Mercader». Best Sellers, Washington, 10-1-1973, p. 296.

\section{Sender, Ramón J.: A man's place}

«Ramón J. Sender. A man's place». The New Yorker, New York, 11-2-1940, p. 86.

ADAMS, Mildred. «The boy grows older». The New York Times, New York, 19-1-1958, p. 5.

BARRY, Griffin. «Ramón J. Sender. A man's place». The New Republic, New York, 2-31941, p. 157.

BATES, Ralph. «Aragon Village». The Nation, New York, 11-2-1940, p. 424-426.

BLACKBURN, Paul. «Ramón J. Sender. Before Noon». The Nation, New York, 19-41958 , p. 346.

BOYD, Ernest. «Ramón J. Sender. A man's place». Saturday Review of Literature, New York, 21-12-1940, p. 11. 
HONIG, Edwin. «Ramón J. Sender. Before Noon». Saturday Review, New York, 4-121958, p. 25.

KAZIN, Alfred. «Ramón J. Sender. A man’s place». New York Herald Tribune, New York, 11-3-1940, p. 3.

LITTEL, Robert. «Ramón J. Sender. A man's place». The Yale Review, Boston, Winter1941, p. 11.

MARSH, F. T. «Ramon Sender's novel of old Aragon». The New York Times, New York, 11-3-1940, p. 8.

RANKIN, R. B. «Ramón J. Sender. A man's place». Library Journal, New York, 15-101940 , p. 874.

W. K. YOUNG. «Ramón J. Sender. Before Noon». San Francisco Chronicle, San Francisco, 16-2-1958, p. 26.

WEST, Anthony. «Ramón J. Sender. Before Noon». The New Yorker, New York, 19-41958 , p. 147.

\section{Sender, Ramón J.: Chronicle of dawn}

«Ramón J. Sender. Chronicle of dawn». The New Yorker, New York, 26-2-1944, p. 86.

BATES, Ralph. «Ramón J. Sender. Chronicle of dawn». Saturday Review of Literature, New York, 15-4-1944, p. 26.

FARBER, Marjorie. «Childhood of a Spanish martyr». The New York Times, New York, 20-2-1944, p. 4.

ROSENFELD, Isaac. «Ramón J. Sender. Chronicle of dawn». The New Republic, New York, 24-4-1944, p. 572.

TRILLING, Diana. «Ramón J. Sender. Chronicle of dawn». The Nation, New York, 18-31944, p. 342.

WOLFE, B. D. «Ramón J. Sender. Chronicle of dawn». New York Herald Tribune, New York, 3-12-1944, p. 3.

\section{Sender, Ramón J.: Dark Wedding}

«Ramón J. Sender. Dark Wedding». The New Yorker, New York, 27-3-1943, p. 71.

GORMAN, Herbert. «Caribbean revolt». The New York Times, New York, 28-4-1943, pp. 10-12.

KAZIN, Alfred. «Ramón J. Sender. Dark Wedding». The New Republic, New York, 4-51943, p. 451

KENNEDY, Leo. «Ramón J. Sender. Dark wedding». Book Week, New York, 4-11-1943, p. 3.

REDMAN, B. R. «Ramón J. Sender. Dark wedding». Saturday Review of Literature, New York, 15-5-1943, p. 13.

SCHORER, Mark. «Ramón J. Sender. Dark wedding». The Yale Review, Boston, Summer1943, p. vi.

SOSKIN, William. «Ramón J. Sender. Dark Wedding». New York Herald Tribune, New York, 28-3-1943, p. 4.

TRILLING, Lionel. «The lower depths». The Nation, New York, 24-4-1943, pp. 602-604.

\section{Sender, Ramón J.: Tales of Cibola}

MYERS, Oliver T. «To die in New Mexico». The Nation, New York, 17-1-1966, pp. 75-76.

\section{Sender, Ramón J.: The affable hangman}

CORKE, Hilary. «Ramón J. Sender. The affable hangman». The New Republic, New York, 30-11-1963, p. 16. 
CURLEY, Thomas. «Ramón J. Sender. The affable hangman». The New York Times Book Review, New York, 9-8-1963, p. 5.

GRAY, James. «Ramón J. Sender. The affable hangman». Saturday Review, New York, 97-1963, p. 22.

MILLER, Warren. «Ramón J. Sender. The affable hangman». Book Review, New York, 299-1963, p. 6.

PEÑALOSA, Fernando. «Ramón J. Sender. The affable hangman». Library Journal, New York, 11-1-1963, p. 4238.

\section{Sender, Ramón J.: The King and the Queen}

«Ramón J. Sender. The King and the Queen». The New Yorker, New York, 29-5-1948, p. 77.

«Ramón J. Sender. The King and the Queen». San Francisco Chronicle, San Francisco, $7-$ 4-1948, p. 17.

BARR, Donald. «The Duchess and her gardener». The New York Times, New York, 27-61948 , p. 4.

BUTCHER, Fanny. «Realistic and mystic blend in R. Sender». Chicago Daily Tribune, Chicago, 6-6-1948, p. 6.

MAYBERRY, George. «Ramón J. Sender. King and the Queen». The New Republic, New York, 31-5-1948, p. 28.

WOLFE, B. D. «Ramón J. Sender. The King and the Queen». New York Herald Tribune, New York, 16-5-1948, p. 5.

\section{Sender, Ramón J.: The Sphere}

«Ramón J. Sender. The Sphere». The New Yorker, New York, 21-5-1949, p. 113.

BROWN, C. M. «Ramón J. Sender. The Sphere». Saturday Review of Literature, New York, 6-4-1949, p. 28.

D. H. «Ramón J. Sender. The Sphere». San Francisco Chronicle, San Francisco, 16-61949, p. 13.

KRIEGER, Murray. «Ramón J. Sender. The Sphere». The Christian Science Monitor, Boston, 24-5-1949, p. 14.

OSTERHOUT, Hilda. «An experiment in symbolism». The New York Times, New York, 5-1-1949, p. 6.

\section{Vilallonga, José Luis de: Man of blood}

«José Luis de Vilallonga. Man of blood». The New Yorker, New York, 25-6-1960, p. 104.

BYAM, M. S. «José Luis de Vilallonga. Man of blood». Library Journal, New York, 31-1960, p. 989.

HALL, Vernon. «José Luis de Vilallonga. Man of blood». New York Herald Tribune, New York, 22-5-1960, p. 9.

RODMAN, Selden. «Lifetime of trials». The New York Times Book Review, New York, 410-1960, p. 42.

SENDER, Ramón. «José Luis de Vilallonga. Man of blood». Saturday Review, New York, 6-11-1960, p. 21.

SOLNER, Ruth. «Loyalist side of Spain's Civil War riles Franco». Los Angeles Times, Los Angeles, 22-5-1960, p. 7.

Vilallonga, José Luis de: The pleasure seeker

LEVIN, Martin. «José Luis de Vilallonga. The pleasure seeker». The New York Times, New York, 17-6-1962, p. 216. 
PEÑALOSA, Fernando. «Ramón J. Sender. The affable hangman». Library Journal, New York, 11-1-1963, p. 4238.

\section{Sender, Ramón J.: The King and the Queen}

«Ramón J. Sender. The King and the Queen». The New Yorker, New York, 29-5-1948, p. 77.

«Ramón J. Sender. The King and the Queen». San Francisco Chronicle, San Francisco, 74-1948, p. 17.

BARR, Donald. «The Duchess and her gardener». The New York Times, New York, 27-61948, p. 4.

BUTCHER, Fanny. «Realistic and mystic blend in R. Sender». Chicago Daily Tribune, Chicago, 6-6-1948, p. 6.

MAYBERRY, George. «Ramón J. Sender. King and the Queen». The New Republic, New York, 31-5-1948, p. 28.

WOLFE, B. D. «Ramón J. Sender. The King and the Queen». New York Herald Tribune, New York, 16-5-1948, p. 5.

\section{Sender, Ramón J.: The Sphere}

«Ramón J. Sender. The Sphere». The New Yorker, New York, 21-5-1949, p. 113.

BROWN, C. M. «Ramón J. Sender. The Sphere». Saturday Review of Literature, New York, 6-4-1949, p. 28.

D. H. «Ramón J. Sender. The Sphere». San Francisco Chronicle, San Francisco, 16-61949, p. 13.

KRIEGER, Murray. «Ramón J. Sender. The Sphere». The Christian Science Monitor, Boston, 24-5-1949, p. 14.

OSTERHOUT, Hilda. «An experiment in symbolism». The New York Times, New York, 5-1-1949, p. 6.

\section{Vilallonga, José Luis de: Man of blood}

«José Luis de Vilallonga. Man of blood». The New Yorker, New York, 25-6-1960, p. 104. BYAM, M. S. «José Luis de Vilallonga. Man of blood». Library Journal, New York, 31-1960, p. 989.

HALL, Vernon. «José Luis de Vilallonga. Man of blood». New York Herald Tribune, New York, 22-5-1960, p. 9.

RODMAN, Selden. «Lifetime of trials». The New York Times Book Review, New York, 410-1960, p. 42.

SENDER, Ramón. «José Luis de Vilallonga. Man of blood». Saturday Review, New York, 6-11-1960, p. 21.

SOLNER, Ruth. «Loyalist side of Spain's Civil War riles Franco». Los Angeles Times, Los Angeles, 22-5-1960, p. 7.

\section{Vilallonga, José Luis de: The pleasure seeker}

LEVIN, Martin. «José Luis de Vilallonga. The pleasure seeker». The New York Times, New York, 17-6-1962, p. 216. 